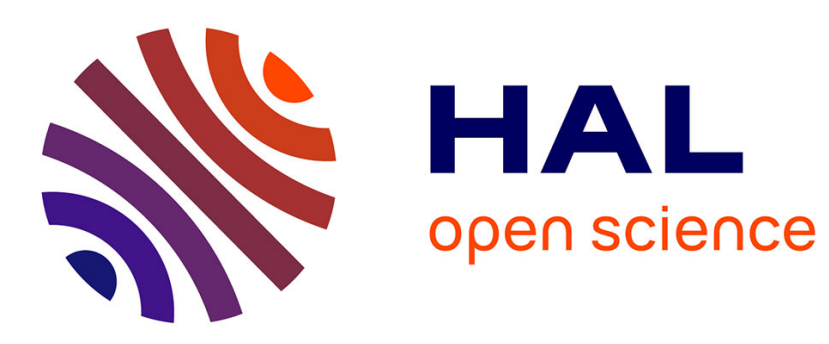

\title{
Dissipative Hyperbolic Systems: the Asymptotic Behavior of Solutions
}

Stefano Bianchini, Bernard Hanouzet, Roberto Natalini

\section{To cite this version:}

Stefano Bianchini, Bernard Hanouzet, Roberto Natalini. Dissipative Hyperbolic Systems: the Asymptotic Behavior of Solutions. Hyperbolic Problems: Theory, Numerics, Applications, Jul 2006, Lyon, France. pp.59-73. hal-00281938

\section{HAL Id: hal-00281938 \\ https://hal.science/hal-00281938}

Submitted on 26 May 2008

HAL is a multi-disciplinary open access archive for the deposit and dissemination of scientific research documents, whether they are published or not. The documents may come from teaching and research institutions in France or abroad, or from public or private research centers.
L'archive ouverte pluridisciplinaire HAL, est destinée au dépôt et à la diffusion de documents scientifiques de niveau recherche, publiés ou non, émanant des établissements d'enseignement et de recherche français ou étrangers, des laboratoires publics ou privés. 


\title{
Dissipative Hyperbolic Systems: the Asymptotic Behavior of Solutions
}

\author{
Stefano Bianchini ${ }^{1}$, Bernard Hanouzet ${ }^{2}$, and Roberto Natalini ${ }^{3}$ \\ 1 SISSA-ISAS, Via Beirut 2-4, I-34014 Trieste, Italy bianchin@sissa.it \\ 2 Mathématiques Appliquées de Bordeaux, Université Bordeaux 1, 351 cours de la \\ Libération, F-33405 Talence, France Bernard.Hanouzet@math.u-bordeaux1.fr \\ 3 Istituto per le Applicazioni del Calcolo "Mauro Picone", Consiglio Nazionale \\ delle Ricerche r.natalini@iac.cnr.it
}

\section{Introduction}

In this talk we shall review some recent results about qualitative beahavior of smooth solutions to the Cauchy problem for general hyperbolic symmetrizable $m$-dimensional systems of balance laws of the form

$$
u_{t}+\sum_{\alpha=1}^{m}\left(f_{\alpha}(u)\right)_{x_{\alpha}}=g(u)
$$

with the initial condition

$$
u(x, 0)=u_{0}(x),
$$

where $u=\left(u_{1}, u_{2}\right) \in \Omega \subseteq \mathbb{R}^{n_{1}} \times \mathbb{R}^{n_{2}}$, with $n_{1}+n_{2}=n$. We assume that there are $n_{1}$ conservation laws in the system, namely that we can take

$$
g(u)=\left(\begin{array}{c}
0 \\
q(u)
\end{array}\right), \text { with } q(u) \in \mathbb{R}^{n_{2}} .
$$

As well known, even for nice initial data, smooth solutions may break down in finite time, due to the appearance of singularities. On the other hand, sometimes dissipative mechanisms induced by the source term can prevent the formation of singularities, at least for small initial data, as for instance for the compressible Euler equations with damping [Ni78, STW03].

Recently, it was proposed in [HN03] a quite general framework of sufficient conditions to have the global existence in time of smooth solutions. Actually, for systems which are endowed with a strictly convex entropy function $\mathcal{E}=\mathcal{E}(u)$, a first natural assumption is the entropy dissipation condition, see [CLL94]. Unfortunately, it is easy to see that this condition is too weak to prevent the formation of singularities. A quite natural supplementary condition can be imposed to entropy dissipative systems, following the classical 
approach by Shizuta and Kawashima [Kw87, SK85]. It is possible to prove that this condition, which is satisfied in many interesting examples, is also sufficient to establish a general result of global existence for small perturbations of equilibrium constant states, see [HN03, Yo04]. These results will be shortly reviewed in Section 2.

Here, we intend to present a short survey of the new results of [BRS06], about the asymptotic behavior in time of the global solutions, then always assuming the existence of a strictly convex entropy and the Shizuta and Kawashima condition. However, let us recall that, starting from the seminal paper by T.P. Liu [Li87], there were some previous studies on $2 \times 2$ systems, see [Na99] for some references. For more general models, we recall [Ze99], for a model of gas dynamics in thermal nonequilibrium, and [RS02], about stability of constant equilibrium states for general hyperbolic systems in one space dimension, under zero-mass perturbations.

The paper is organized as follows: Section 2 is devoted to recall some basic results about hyperbolic systems with entropy dissipation and the ShizutaKawashima condition. In this section we also introduce the decomposition of the linearized system, which will be called the Conservative-Dissipative form, which will be necessary to cast the decay results in the optimal way. Section 3 contains some precise results about the asymptotic behavior of the Green kernel for the linearized problem. Finally, Section 4 is devoted to the study of the decay properties of the nonlinear system. Not only we shall present the decay results for both the conservative and the dissipative part of the solution, but we shall show also that the conservative variable approaches the conservative part of the solution of the corresponding linearized problem, faster that the decay of the heat kernel for $m \geq 2$. Also, it is possible to see that the solution of the parabolic problem given by the Chapman-Enskog expansion, is a good approximation of the conservative part of the solution of the nonlinear hyperbolic system. For $m \geq 2$ the Chapman-Enskog operator is linear, while, in one space dimension, the decay of the nonlinear part has a stronger influence, and so we can only show the faster convergence towards the solution of a parabolic equation with quadratic nonlinearity. Let us point out that the present results can be useful to design more accurate numerical approximations of these problems, which are increasingly accurate for large times, see $[\mathrm{ABN} 06]$ for some preliminary results in this direction.

Finally, we notice again that these results were obtained by assuming all the time the condition (SK). Unfortunately, this condition is not satisfied by many models, as for instance for the model studied in [Ze99], where however global existence of solutions has been established. For the Kerr-Debye system, studied in [HH00, CH106, CH206], condition (SK) is not always verified for $m=1$, see [HN03], and never verified for $m \geq 2$. 


\section{A short review of some basic structures for the entropy dissipative hyperbolic systems}

In the following we shall consider a general $m$-dimensional system of balance laws given by equation (1), with the source term $g=g(u)$ given by (3). According to the general theory of hyperbolic systems of conservation laws [Da00], we shall assume that the system satisfies an entropy principle: there exists a strictly convex function $\mathcal{E}=\mathcal{E}(u)$, the entropy density, and some related entropy-flux functions $\mathcal{F}_{\alpha}=\mathcal{F}_{\alpha}(u)$, such that for every smooth solution $u \in \Omega$ to system (1), there holds

$$
\mathcal{E}(u)_{t}+\sum_{\alpha=1}^{m}\left(\mathcal{F}_{\alpha}(u)\right)_{x_{\alpha}}=\mathcal{G}(u)
$$

where $\mathcal{F}_{\alpha}{ }^{\prime}=\left(f_{\alpha}^{\prime}\right)^{T} \mathcal{E}^{\prime}$ and $\mathcal{G}=\mathcal{E}^{\prime} \cdot g$. Let us introduce the set $\gamma$ of the equilibrium points:

$$
\gamma=\{u \in \Omega ; g(u)=0\}
$$

We shall assume that system (1) is non-degenerate in the sense that around a given $\bar{u} \in \gamma$, it holds

$$
q_{u_{2}}(\bar{u}) \text { is non singular. }
$$

We shall also assume that system (1) is entropy dissipative around the given equilibrium point $\bar{u} \in \gamma$ and $u \in \Omega$, in the sense that we have

$$
\left(\mathcal{E}^{\prime}(u)-\mathcal{E}^{\prime}(\bar{u})\right) \cdot g(u) \leq 0 .
$$

Following [Go61, LF71], it is now possible to symmetrize our system by introducing a new variable, the entropy variable, which is just given by $W=\mathcal{E}^{\prime}(u)$. This change of variable is very useful in the statement of the global existence results. Actually, since $\mathcal{E}$ is a strictly convex function, we can inverse $\mathcal{E}^{\prime}$ to recover the original variable $u$ by the inverse map $\Phi \doteq\left(\mathcal{E}^{\prime}\right)^{-1}$. Let us set now $A_{0}(W) \doteq \Phi^{\prime}(W), C_{\alpha}(W) \doteq D f_{\alpha}(\Phi(W)) A_{0}(W)$, and $G(W)=g(\Phi(W))=$ $\left(\begin{array}{c}0 \\ Q(W)\end{array}\right)$. It is easy to see that the matrix $A_{0}(W)$ is symmetric positive definite and, for every $\alpha=1 \ldots, m, C_{\alpha}(W)$ is symmetric. Then, selecting $W$ as the new variable, our system reads

$$
A_{0}(W) W_{t}+\sum_{\alpha=1}^{m} C_{\alpha}(W) W_{x_{\alpha}}=G(W) .
$$

In the following, without loss of generality, we can always suppose $\bar{u}=0 \in \gamma$ and consider system (1) with $g(0)=0, f_{\alpha}(0)=0$, and endowed with a quadratic entropy function $\mathcal{E}$. Next, we focus our investigation on a slightly restricted class of entropy dissipative non-degenerate systems, namely the systems such that 


$$
Q(W)=D(W) W_{2} \text {, with } D(0) \text { negative definite. }
$$

To continue our analysis of smooth solutions for dissipative hyperbolic systems, we need some supplementary coupling conditions to avoid shock formation. A very natural condition was first introduced by Shizuta and Kawashima in [SK85], for hyperbolic-parabolic systems. Let us introduce this condition for the original unknown, i.e. for system (1).

Definition 1. The system (1) verifies condition (SK), if every eigenvector of $\sum_{\alpha=1}^{m} D f_{\alpha}(0) \xi_{\alpha}$ is not in the null space of $D g(0)$, for every $\xi \in \mathbb{R}^{m} \backslash\{0\}$.

About the existence of a solution, we recall the following result [HN03, Yo04].

Theorem 1. Assume that system (1) is strictly entropy dissipative and condition (SK) is satisfied. Then there exists $\delta>0$ such that, if $\left\|u_{0}\right\|_{s} \leq \delta$, with $s \geq[m / 2]+2$, there is a unique global solution $u$ of (1)-(2), which verifies

$$
u \in C^{0}\left([0, \infty) ; H^{s}\left(\mathbb{R}^{m}\right)\right) \cap C^{1}\left([0, \infty) ; H^{s-1}\left(\mathbb{R}^{m}\right)\right),
$$

and such that, in terms of the entropy variable $W=\left(W_{1}, W_{2}\right)$,

$$
\sup _{0 \leq t<+\infty}\|W(t)\|_{s}^{2}+\int_{0}^{+\infty}\left(\left\|\nabla W_{1}(\tau)\right\|_{s-1}^{2}+\left\|W_{2}(\tau)\right\|_{s}^{2}\right) d \tau \leq C(\delta)\left\|W_{0}\right\|_{s}^{2},
$$

where $C(\delta)$ is a positive constant.

Let us consider now the linearized version of system (1), namely, setting $A_{\alpha}=D f_{\alpha}(0)$ and $B=D g(0)$,

$$
u_{t}+\sum_{\alpha=1}^{m} A_{\alpha} u_{x_{\alpha}}=B u, \quad u \in \mathbb{R}^{n}, x \in \mathbb{R}^{m}, t \in \mathbb{R}^{+},
$$

with $B$ of the form

$$
B=\left[\begin{array}{cc}
0 & 0 \\
D_{1} & D_{2}
\end{array}\right], \quad D_{1} \in \mathbb{R}^{n_{1} \times n_{2}}, D_{2} \in \mathbb{R}^{n_{2} \times n_{2}},
$$

with $n=n_{1}+n_{2}$. Set $A(\xi)=\sum_{\alpha=1}^{m} A_{\alpha} \xi_{\alpha}$. According to the previous discussion, we can assume that

(H1) there is a symmetric positive definite matrix $A_{0}$ such that $A_{\alpha} A_{0}$ is symmetric, for every $\alpha=1, \ldots, m$, and

$$
B A_{0}=\left[\begin{array}{ll}
0 & 0 \\
0 & D
\end{array}\right]
$$

where $D \in \mathbb{R}^{n_{2} \times n_{2}}$ is negative definite;

(H2) any eigenvector of $A(\xi)$ is not in the null space of $B$, for every $\xi \in \mathbb{R}^{m} \backslash\{0\}$. 
To give a precise picture of the asymptotic behavior of the solutions for large times, we have to transform the original system in a suitable form, using a special projection of the original variables.

Definition 2. The partially dissipative system (10) is in Conservative-Dissipative form ( $C$ - $D$ form) if the matrices $A_{\alpha}$ are symmetric and there exists a negative definite matrix $D \in \mathbb{R}^{n_{2} \times n_{2}}$, such that

$$
B=\left(\begin{array}{ll}
0 & 0 \\
0 & D
\end{array}\right)
$$

In the following $w_{1} \doteq w_{c}$ is called the conservative variable, while $w_{2} \doteq w_{d}$ is the dissipative one.

We shall prove in the following that there exists a linear change of variable such that (10) takes the C-D form under assumptions (H1). We shall construct the C-D variables using the projection $Q_{0}$ on the null space and the complementing projection $Q_{-}=I-Q_{0}$. We can compute $Q_{0}$ by using the classical representation formula, which yields

$$
Q_{0}=A_{0}^{1 / 2}\left[\begin{array}{cr}
\left(A_{0,11}\right)^{-1} & 0 \\
0 & 0
\end{array}\right] A_{0}^{1 / 2} .
$$

Note that, due to the assumptions on $A_{0}$, this projector is symmetric. In particular we can choose left and right projectors $L_{0} \in \mathbb{R}^{n \times n_{1}}, R_{0} \in \mathbb{R}^{n_{1} \times n}$, so that $Q_{0}=R_{0} L_{0}, L_{0} R_{0}=I \in \mathbb{R}^{n_{1} \times n_{1}}, L_{0}=R_{0}^{T}$, and they are uniquely given by

$$
R_{0}=A_{0}^{1 / 2}\left[\begin{array}{c}
\left(A_{0,11}\right)^{-1 / 2} \\
0
\end{array}\right], \quad L_{0}=\left[\left(A_{0,11}\right)^{-1 / 2} 0\right] A_{0}^{1 / 2} .
$$

We define the complementary projection $Q_{-}$such that $Q_{-} \doteq I-Q_{0}=R_{-} L_{-}$, $L_{-} R_{-}=I \in \mathbb{R}^{n_{2} \times n_{2}}, L_{-}=R_{-}^{T}$. The matrices $R_{-}, L_{-}$are uniquely given by

$$
R_{-}=A_{0}^{-1 / 2}\left[\left(\begin{array}{c}
0 \\
\left(\left(A_{0}^{-1}\right)_{22}\right)^{-1 / 2}
\end{array}\right], \quad L_{-}=\left[0\left(\left(A_{0}^{-1}\right)_{22}\right)^{-1 / 2}\right] A_{0}^{-1 / 2} .\right.
$$

If we set $w_{1}=L_{0} A_{0}^{-1 / 2} u$ and $w_{2}=L_{-} A_{0}^{-1 / 2} u$, we obtain the following result.

Proposition 1. If $u$ is a solution to system (10), then, under assumption (H1),

$$
w=M u=\left[\begin{array}{cc}
\left(A_{0,11}\right)^{-1 / 2} & 0 \\
\left(\left(A_{0}^{-1}\right)_{22}\right)^{-1 / 2}\left(A_{0}^{-1}\right)_{21} & \left(\left(A_{0}^{-1}\right)_{22}\right)^{1 / 2}
\end{array}\right] u
$$

is a solution of a system in $C-D$ form.

In the following, we are always going to assume that the unknown $u$ is chosen in such a way that (10) is in conservative-dissipative form. In this case, we say that also system (1) is in conservative-dissipative form and we shall set $u=\left(u_{c}, u_{d}\right) \in \mathbb{R}^{n_{1}} \times \mathbb{R}^{n_{2}}$. 
Example 1. The $p$-system with relaxation. Let us consider system

$$
\left\{\begin{array}{l}
\partial_{t} u+\partial_{x} v=0 \\
\partial_{t} v+\partial_{x} \sigma(u)=h(u)-v
\end{array}\right.
$$

with $\sigma^{\prime}(u)>0$. One symmetrizer for the linearized version of (16) is given by $A_{0}=\left(\begin{array}{cc}1 & a \\ a & \lambda^{2}\end{array}\right)$, with $\lambda=\sqrt{\sigma^{\prime}(0)}$ and $a=h^{\prime}(0)$. The matrix $A_{0}$ is positive definite if it holds the subcharacteristic condition $\lambda>|a|$. It is easy to verify that assumption (H1) is verified and the proper C-D form of the system is found for the new unknowns

$$
\left(\begin{array}{l}
u_{c} \\
u_{d}
\end{array}\right)=M\left(\begin{array}{l}
u \\
v
\end{array}\right)=\left(\begin{array}{cc}
1 & 0 \\
-a\left(\lambda^{2}-a^{2}\right)^{-\frac{1}{2}} & \left(\lambda^{2}-a^{2}\right)^{-\frac{1}{2}}
\end{array}\right)\left(\begin{array}{l}
u \\
v
\end{array}\right)
$$

see formula (2.29) in [BRS06].

\section{The Green kernel for linear dissipative systems}

\subsection{The one dimensional case}

We want to characterize the Green kernel $\Gamma(t)$ for a linear dissipative hyperbolic system. The fact that we are in dimension one will help us in inverting the Fourier transforms, hence giving explicit form to the principal parts of $\Gamma(t)$. We can consider directly a system in C-D form, according to the results of Section 2. So we write our system as

$$
w_{t}+A w_{x}=B w,
$$

where $w=\left(w_{c}, w_{d}\right) \in \mathbb{R}^{n_{1}} \times \mathbb{R}^{n_{2}}$. We assume that the matrix $A$ is symmetric and the matrix $B$ verifies (12). We assume also that (17) verifies condition (SK). We want to present a precise description of the Green kernel $\Gamma(t, x)$ of (17), which is the solution to problem

$$
\left\{\begin{array}{l}
\Gamma_{t}+A \Gamma_{x}=B \Gamma \\
\Gamma(0, x)=\delta(x) I
\end{array}\right.
$$

by extending the approach used in [Ze99].

Consider the entire function $E(z)=B-z A$. The Fourier transform of the solution to (18) is given by $\hat{\Gamma}(t, \xi)=e^{E(i \xi) t}$. Notice that also $\hat{\Gamma}(t, z)=e^{E(z) t}$ is an entire function of $z$. In general, if $z$ is not an exceptional point, the function $E(z)$, is represented by 


$$
E(z)=\sum_{j} \lambda_{j}(z) P_{j}(z)+\sum_{j} D_{j}(z)
$$

where $\lambda_{j}$ are the eigenvalues of $E(z), P_{j}(z)$ the corresponding eigenprojections and $D_{j}$ are the nilpotent matrices, due to the fact that in general $E$ is not diagonalizable, since we are not assuming that the matrix $B$ is symmetric. We now study which consequences have the assumptions (H1), (H2) on $E(z)$ and $\hat{\Gamma}(t, z)$ near the point $z=0$ and $z=\infty$. Both points are in general exceptional. For $z \rightarrow 0, n_{1}$ eigenvalues different from 0 converges to 0 . When $|z| \rightarrow \infty$, the matrix $A$ is diagonalizable, but it can have common eigenvalues: then the perturbation $B / z$ will in general remove part of this degeneracy.

First we consider the case near $z=0$. It has been shown in [BRS06], by standard representation formula, that we can expand the projector in the following way:

$$
P(z)=\left[\begin{array}{cc}
I+\mathcal{O}\left(z^{2}\right) & z A_{12} D^{-1}+\mathcal{O}\left(z^{2}\right) \\
z D^{-1} A_{21}+\mathcal{O}\left(z^{2}\right) & z^{2} D^{-1} A_{21} A_{12} D^{-1}+\mathcal{O}\left(z^{3}\right)
\end{array}\right] .
$$

We introduce the right and left eigenprojectors of $P(z), R(z) \in \mathbb{R}^{n \times n_{1}}, L(z) \in$ $\mathbb{R}^{n_{1} \times n}$, which verify $P(z)=R(z) L(z), L(z) R(z)=I$. We can find the power series of $L(z)$ and $R(z): L(z)=L_{0}+z L_{1}+z^{2} L_{1}+\mathcal{O}\left(z^{3}\right)$, and $R(z)=L_{0}+$ $z R_{1}+z^{2} R_{1}+\mathcal{O}\left(z^{3}\right)$. We compute $L_{0}$ and $R_{0}$ by

$$
L_{0}=R_{0}^{T}=\left[\begin{array}{ll}
I_{n_{1}} & 0
\end{array}\right]
$$

The following terms can be also computed explicitely. So, we can decompose $E(z)$ according to the right and left operators:

$$
E(z)=R(z) F(z) L(z)+E_{1}(z)
$$

where $F(z) \doteq L(z) E(z) R(z) \in \mathbb{R}^{n_{1} \times n_{1}}$ and $E_{1}$ is a rest with a faster decay. We have

$$
\begin{aligned}
F(z) & =\left(L_{0}+z L_{1}+\mathcal{O}\left(z^{2}\right)\right)(B-z A)\left(R_{0}+z R_{1}+\mathcal{O}\left(z^{2}\right)\right) \\
& =-z A_{11}-z^{2} A_{12} D^{-1} A_{21}+\mathcal{O}\left(z^{3}\right) .
\end{aligned}
$$

The matrix $A_{11}$ is symmetric, from assumption (H1), so that we can write for some eigenvalues $\lambda_{j}^{1}$, with multiplicity $m_{j}^{\prime}$, and left and right eigenprojections $l_{j} \in \mathbb{R}^{m_{j}^{\prime} \times n_{1}}, r_{j} \in \mathbb{R}^{n_{1} \times m_{j}^{\prime}}$, with $l_{j}=r_{j}^{T}$,

$$
A_{11}=\sum_{j=1}^{m^{\prime}} \lambda_{j}^{1} r_{j} l_{j}
$$

By a further decomposition, we obtain, for some projectors $P_{j k}$ and $D_{j k}$, the projection of $E(z)$ on the null eigenvalue as

$$
R(z) F(z) L(z)=\sum_{j k}\left(-z \lambda_{j}^{1} I-z^{2} c_{j k} I+\mathcal{O}\left(z^{3}\right)\right) P_{j k}(z)+D_{j k}(z) .
$$


Proposition 2. We have the following decomposition near $z=0$

$$
E(z)=\sum_{j k}\left(\Lambda_{j k}(z) P_{j k}(z)+D_{j k}(z)\right)+E_{1}(z),
$$

where the $\Lambda_{j k}$ are diagonal $n \times n$ matrices composed by the $n_{1}$ eigenvalues $\lambda_{j k}$, the coefficients $c_{j k}$ having strictly negative real part, thanks to assumption (H2).

We can perform a similar, but simpler, analysis for $|z| \rightarrow \infty$, since we can write

$$
E(z)=B-z A=z\left(-A+\frac{1}{z} B\right)=z \tilde{E}(1 / z),
$$

where $\tilde{E}(\zeta)=-A+\zeta B$.

Proposition 3. We have the following decomposition near $z=\infty$

$$
E(z)=\sum_{j k}\left(\Upsilon_{j k}(z) \mathcal{P}_{j k}(z)+\mathcal{D}_{j k}(z)\right)
$$

where $\Upsilon_{j k}$ is the diagonal matrix whose entries are the eigenvalues of the $j k$ family, the coefficients $b_{j k}$ having strictly negative real part, thanks to assumption (H2).

Now, we are ready to estimate the global behavior for large $t$ of the Green kernel $\Gamma(t, x)$ using the local expansions contained in Propositions 2 and 3. We associate a diffusive operator with Green function $K(t, x)$ to the expansion (26), and a dissipative transport operator with Green function $\mathcal{K}(t, x)$ to $(27)$, and we estimate the remainder term $R(t, x)=\Gamma(t, x)-K(t, x)-\mathcal{K}(t, x)$. In the following we shall consider the Green kernel as composed of 4 parts, acting on $w_{c}, w_{d}$ :

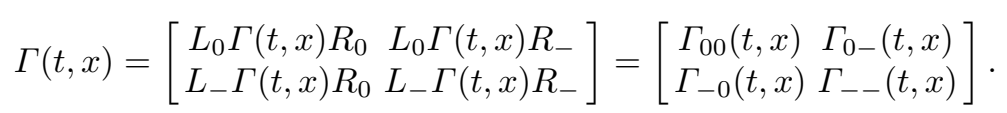

We can associate to each term of $P_{j k}$ in (25), the solution $g_{j k}$ to the parabolic equation

$$
w_{t}+\lambda_{j}^{1} w_{x}=-\left(c_{j k} I+d_{j k}\right) w_{x x}, \quad w \in \mathbb{R}^{m_{j}^{\prime}},
$$

where $c_{j k}=-\mu_{j k}-i \nu_{j k}$, with $\mu_{j k}>0$. The solution $g_{j k}$ can be computed explicitly, and we have in any case that for some $c>0$

$$
\left|g_{j k}(t, x)\right| \leq \frac{\mathcal{O}(1)}{\sqrt{t}} e^{-\left(x-\lambda_{j}^{1} t\right)^{2} /(c t)} \quad \forall k,(t, x) \in \mathbb{R}^{+} \times \mathbb{R} .
$$

The function

$$
K(t, x) \doteq \sum_{j k}\left[\begin{array}{cc}
r_{j}\left(g_{j k}(t, x) p_{j k}\right) r_{j}^{T} & -r_{j}\left(\frac{d g_{j k}}{d x} p_{j k}\right) r_{j}^{T} A_{12} D^{-1} \\
-D^{-1} A_{21} r_{j}\left(\frac{d g_{j k}}{d x} p_{j k}\right) p_{j k} r_{j}^{T} & D^{-1} A_{21} r_{j}\left(\frac{d^{2} g_{j k}}{d x^{2}} p_{j k}\right) r_{j}^{T} A_{12} D^{-1}
\end{array}\right]
$$


collects the principal parts of each component (28) of the Green kernel $\Gamma(t, x)$. Near $z=\infty$, we can associate to each projectors, the Fourier transform of the Green kernel of the transport equation

$$
w_{t}+\lambda_{j} w_{x}=\left(b_{j k} I+\tilde{d}_{j k}\right) w, \quad w \in \mathbb{R}^{m_{j k}} .
$$

Since $\Re\left(b_{j k}\right) \leq-c<0$, we can estimate its solution $\tilde{g}_{j k}$ by

$$
\left|\tilde{g}_{j k}(t, x)\right| \leq C \delta\left(x-\lambda_{j} t\right) e^{-c t} \quad \forall k,(t, x) \in \mathbb{R}^{+} \times \mathbb{R} .
$$

We associate to the kernels $\tilde{g}_{j k}$, the hyperbolic Green function

$$
\mathcal{K}(t, x)=\sum_{j k} \delta\left(x-\lambda_{j} t\right) e^{b_{j k} t} e^{t \mathcal{D}_{j k}(\infty)} \mathcal{P}_{j k}(\infty) .
$$

Theorem 2. Let $\Gamma(t, x)$ be the Green function of system (17), under the assumptions (H1) and (H2). Let $K(t, x)$ be the Green function of the diffusive operator given by $(31)$ and $\mathcal{K}(t, x)$ the Green function of the dissipative transport operator given by (34). Then, we have the decomposition

$$
\Gamma(t, x)=K(t, x) \chi\{\underline{\lambda} t \leq x \leq \bar{\lambda} t, t \geq 1\}+\mathcal{K}(t, x)+R(t, x) \chi\{\underline{\lambda} t \leq x \leq \bar{\lambda} t\},
$$

where $\chi$ is the characteristic function, and $\bar{\lambda}:=\max _{j} \lambda_{j}, \underline{\lambda}:=\min _{j} \lambda_{j}$. The matrix $R(t, x)$ can be written as

$$
R(t, x)=\sum_{j} \frac{e^{-\left(x-\lambda_{j}^{1} t\right)^{2} / C t}}{1+t}\left[\begin{array}{cc}
\mathcal{O}(1) & \mathcal{O}(1)(1+t)^{-1 / 2} \\
\mathcal{O}(1)(1+t)^{-1 / 2} & \mathcal{O}(1)(1+t)^{-1}
\end{array}\right] .
$$

where $R(t, x)$ can for some constant $c$. Here $\lambda_{j}^{1}$ are the eigenvalues of the symmetric block $A_{11}$ of the matrix $A$.

\subsection{The multi dimensional Green function}

We want to state an analogous result for multi dimensional systems. We consider the Cauchy problem for the linear relaxation system in the ConservativeDissipative form

$$
\begin{gathered}
w_{t}+\sum_{\alpha=1}^{m} A_{\alpha} w_{x_{\alpha}}=B w, \quad w \in \mathbb{R}^{n_{1}+n_{2}}, \\
w(0, \cdot)=w^{0} .
\end{gathered}
$$

We assume that $A_{\alpha}, \alpha=1, \ldots, m$, are symmetric matrices and we have assumptions (H1) and (H2). Set, for $\xi \in \mathbb{R}^{m}$,

$$
A(\xi):=\sum_{\alpha=1}^{m} \xi_{\alpha} A_{\alpha}, \quad E(i \xi)=B-i A(\xi) .
$$


Let us introduce the polar coordinates in $\mathbb{R}^{m}$, to say $\xi=\rho \zeta$, with $\rho=|\xi|$ and $\zeta \in S^{m-1}$, and set $E(i \rho, \zeta)=E(i \rho \zeta)$. As before, we want to study the Green kernel $\Gamma(t, x)$ of (37). The solution of the Cauchy problem (37)-(38) is given by $w(t, \cdot)=\Gamma(t, \cdot) * w^{0}$. Let us study the expansion of $E(z, \zeta)=$ $B-z A(\zeta)$ near $z=0$. We can use the result of Section 3, noting that the matrix $A$ is simply replaced by $A(\zeta)$. We introduce the total projector $P(z, \zeta)$ corresponding to all the eigenvalues near 0 , and $P_{-}(z, \zeta)=I-P(z, \zeta)$ is the projector corresponding to the whole family of the eigenvalues with strictly negative real part. As in (23), we have

$$
\begin{gathered}
F(z, \zeta) \doteq L(z, \zeta) E(z, \zeta) R(z, \zeta)=-z A_{11}(\zeta)-z^{2} A_{12}(\zeta) D^{-1} A_{21}(\zeta)+\mathcal{O}\left(z^{3}\right) \\
F_{-}(z) \doteq L_{-}(z, \zeta) E(z, \zeta) R_{-}(z, \zeta)=D-z A_{22}(\zeta)+\mathcal{O}\left(z^{2}\right) .
\end{gathered}
$$

So we make a decomposition of the Green operator:

$$
\Gamma(t)=K(t)+\mathcal{K}(t),
$$

where, for a given constant $a$ small enough,

$$
\begin{gathered}
\widehat{K}(t) \doteq \chi(|\xi| \leq a) R(i \xi) e^{F(i \xi) t} L(i \xi) \\
\widehat{\mathcal{K}}(t) \doteq \chi(|\xi| \leq a) R_{-}(i \xi) e^{F_{-}(i \xi) t} L_{-}(i \xi)+\chi(|\xi|>a) e^{E(i \xi) t} .
\end{gathered}
$$

Theorem 3. Consider the linear PDE in the Conservative-Dissipative form

$$
w_{t}+\sum_{\alpha=1}^{m} A_{\alpha} w_{x_{\alpha}}=B w,
$$

where $A_{\alpha}, B$ satisfy the assumption (SK) of Definition 1, and let $Q_{0}=R_{0} L_{0}$, $Q_{-}=I-Q_{0}=R_{-} L_{-}$be the eigenprojectors on the null space and the negative definite part of $B$. Then, for any function $w^{0} \in L^{1} \cap L^{2}\left(\mathbb{R}^{m}, \mathbb{R}^{n}\right)$, the solution $w(t)=\Gamma(t) w^{0}$ of (37), (38) can be decomposed as

$$
w(t)=\Gamma(t) w^{0}=K(t) w^{0}+\mathcal{K}(t) w^{0},
$$

where the following estimates hold: for any multi index $\beta$ and for every $p \in$ $[2,+\infty]$,

$$
\begin{aligned}
& \left\|L_{0} D^{\beta} K(t) w^{0}\right\|_{L^{p}} \leq C(|\beta|) \min \left\{1, t^{-\frac{m}{2}\left(1-\frac{1}{p}\right)-|\beta| / 2}\right\}\left\|L_{0} w^{0}\right\|_{L^{1}} \\
& +C(|\beta|) \min \left\{1, t^{-\frac{m}{2}\left(1-\frac{1}{p}\right)-1 / 2-|\beta| / 2}\right\}\left\|L_{-} w^{0}\right\|_{L^{1}}, \\
& \left\|L_{-} D^{\beta} K(t) w^{0}\right\|_{L^{p}} \leq C(|\beta|) \min \left\{1, t^{-\frac{m}{2}\left(1-\frac{1}{p}\right)-1 / 2-|\beta| / 2}\right\}\left\|L_{0} w^{0}\right\|_{L^{1}} \\
& +C(|\beta|) \min \left\{1, t^{-\frac{m}{2}\left(1-\frac{1}{p}\right)-1-|\beta| / 2}\right\}\left\|L_{-} w^{0}\right\|_{L^{1}}, \\
& \left\|D^{\beta} \mathcal{K}(t) w^{0}\right\|_{L^{2}} \leq C e^{-c t}\left\|D^{\beta} w^{0}\right\|_{L^{2}} .
\end{aligned}
$$


Example 2. Consider for example the linearized isentropic Euler equations with damping

$$
\left\{\begin{array}{l}
\rho_{t}+\operatorname{div} v=0 \\
v_{t}+\nabla \rho=-v
\end{array}\right.
$$

To fix the ideas, take $m=3, n=4=n_{1}+n_{2}=1+3$. Clearly the system is already in the Conservative-Dissipative form and condition (SK) is satisfied. We decompose $K(t, x)$ as

$$
K(t, x)=\left[\begin{array}{cc}
G(t, x) & (\nabla G(t, x))^{T} \\
\nabla G(t, x) & \nabla^{2} G(t, x)
\end{array}\right]+R_{1}(t, x),
$$

where $G(t, x)$ is the heat kernel for $u_{t}=\Delta u$, and $R_{1}(t, x)$ satisfies the bound

$$
R_{1}(t, x)=\frac{e^{-c|x|^{2} / t}}{(1+t)^{2}}\left[\begin{array}{cc}
\mathcal{O}(1) & \mathcal{O}(1)(1+t)^{-1 / 2} \\
\mathcal{O}(1)(1+t)^{-1 / 2} & \mathcal{O}(1)(1+t)^{-1}
\end{array}\right] .
$$

\section{Decay estimates in the nonlinear case and more accurate asymptotic behavior}

In this section we study the time decay properties of the global smooth solutions to a nonlinear entropy strictly dissipative relaxation system in conservative-dissipative form.

\subsection{Nonlinear decay estimates}

We now prove the decay estimates in $L^{p}\left(\mathbb{R}^{m} ; \mathbb{R}^{n}\right), p \in[2,+\infty]$, for the solution $u$ with initial data in $L^{1} \cap H^{s}$, with $s$ sufficiently large, for the non linear equation

$$
u_{t}+\sum_{\alpha=1}^{m}\left(f_{\alpha}(u)\right)_{x_{\alpha}}=g(u)
$$

with $f_{\alpha}(0)=g(0)=0$ and initial condition

$$
u(x, 0)=u_{0}(x) .
$$

We shall assume that the system (51) is strictly entropy dissipative and condition (SK) is satisfied. Under the assumptions of Theorem 1, we consider the global solution $u$ of (51)-(52).

Theorem 4. Let $u(t)$ be a smooth global solution to problem (51), (52). Let $E_{s}=\max \left\{\|u(0)\|_{L^{1}},\|u(0)\|_{H^{s}}\right\}$, and assume $E_{[m / 2]+2}$ small enough. Let $p \in$ $[2,+\infty]$. The following decay estimate holds 


$$
\left\|D^{\beta} u(t)\right\|_{L^{p}} \leq C \min \left\{1, t^{-\frac{m}{2}\left(1-\frac{1}{p}\right)-|\beta| / 2}\right\} E_{|\beta|+[m / 2]+1},
$$

with $C=C\left(E_{|\beta|+\sigma}\right)$, for $\sigma$ large enough. For $m=1$, this estimate holds for $p \in[1,+\infty]$.

We now study the faster decay of the dissipative variables $u_{d}(t)$.

Theorem 5. Under the assumptions of Theorem 4, we have the following decay estimates for the dissipative part of u:

$$
\left\|D^{\beta} u_{d}(t)\right\|_{L^{p}} \leq C \min \left\{1, t^{-\frac{m}{2}\left(1-\frac{1}{p}\right)-1 / 2-|\beta| / 2}\right\} E_{|\beta|+[m / 2]+1},
$$

with $C=C\left(E_{|\beta|+\sigma}\right)$, for $\sigma$ large enough, and $p \in[2,+\infty]$. For $m=1$, this estimate holds for $p \in[1,+\infty]$.

\subsection{Decay to the linearized profile}

We consider here the difference among the solution of the nonlinear equation (51) and the linearized one

$$
u_{t}+\sum_{\alpha=1}^{m} D f_{\alpha}(0) u_{x_{\alpha}}=\left(\begin{array}{c}
0 \\
D_{u_{d}} q(0) u_{d}
\end{array}\right) .
$$

We can show that, if the dimension $m \geq 2$, then the solution to the nonlinear equation converges to the linearized solution. Using the linear estimates, and with a special argument for the case $m=2$, we have the following result. The following result does not hold for $m=1$.

Theorem 6. Let $u_{l}$ be the solution of problem (55), (52), under the assumptions of Theorem 4, for $m \geq 2$ and $p \in[2, \infty]$, we have the following decay estimate

$$
\left\|D^{\beta}\left(u(t)-u_{l}(t)\right)\right\|_{L^{p}} \leq C \min \left\{1, t^{-\frac{m}{2}\left(1-\frac{1}{p}\right)-|\beta| / 2-1 / 2}\right\} E_{|\beta|+[m / 2]+1},
$$

with $C=C\left(E_{|\beta|+\sigma}\right)$, for $\sigma$ large enough.

\subsection{The Chapman-Enskog expansion}

Next we show how the solutions to the parabolic Chapman-Enskog expansion approximate the conservative part of the solutions to the nonlinear hyperbolic problem (1). We use the conservative-dissipative decomposition of $u$ to yields

$$
u_{c, t}+\sum_{\alpha=1}^{m} A_{\alpha, 11}(0) u_{c, x_{\alpha}}+\sum_{\alpha=1}^{m} A_{\alpha, 12}(0) u_{d, x_{\alpha}}=L_{0} \sum_{\alpha=1}^{m}\left(A_{\alpha}(0) u-f_{\alpha}(u)\right)_{x_{\alpha}} ;
$$




$$
\begin{array}{r}
u_{d, t}+\sum_{\alpha=1}^{m} A_{\alpha, 21}(0) u_{c, x_{\alpha}}+\sum_{\alpha=1}^{m} A_{\alpha, 22}(0) u_{d, x_{\alpha}}=D_{u_{d}} q(0) u_{d} \\
\quad+L_{-} \sum_{\alpha=1}^{m}\left(A_{\alpha}(0) u-f_{\alpha}(u)\right)_{x_{\alpha}}+\left(q(u)-D_{u_{d}} q(0) u_{d}\right) .
\end{array}
$$

Now, consider the linear parabolic equation

$$
w_{t}+\sum_{\alpha=1}^{m} A_{\alpha, 11}(0) w_{x_{\alpha}}+\sum_{\beta=1}^{m} \sum_{\alpha=1}^{m} A_{\alpha, 12}(0)\left(D_{u_{d}} q(0)\right)^{-1} A_{\beta, 21}(0) w_{x_{\alpha} x_{\beta}}=0
$$

and denote by $u_{p}(t)$ the solution of the weakly parabolic equation (59) with

$$
u_{p}(0)=L_{0} u(0) \text {. }
$$

Compute $u_{d}$ using (58) and decay estimates of Theorems 4 and 5 . This yields the following sharper decay result.

Theorem 7. Let $u_{p}$ be the solution of problem (59), (60), under the assumptions of Theorem 4 , for $m \geq 2$ and $p \in[2, \infty]$, we have the following decay estimate

$$
\left\|D^{\beta}\left(u_{c}(t)-u_{p}(t)\right)\right\|_{L^{p}} \leq C \min \left\{1, t^{-\frac{m}{2}\left(1-\frac{1}{p}\right)-|\beta| / 2-1 / 2}\right\} E_{|\beta|+[m / 2]+1},
$$

with $C=C\left(E_{|\beta|+\sigma}\right)$, for $\sigma$ large enough.

Example 3. Consider the isentropic dissipative Euler equations

$$
\left\{\begin{array}{l}
\rho_{t}+\operatorname{div}(\rho v)=0 \\
(\rho v)_{t}+\operatorname{div}(\rho v \otimes v)+\frac{1}{\gamma} \nabla \rho^{\gamma}=-v
\end{array}\right.
$$

We can linearize the system around the constant state $(\bar{\rho}, \bar{v})=(1,0)$, so obtaining system (48) of Example 2. In that case we can immediately apply Theorems 4, 5, 6, and 7. In particular, by eliminating $v$ in (48), we obtain the estimate

$\left\|D^{\beta}\left(\rho(t)-\rho_{w}(t)\right)\right\|_{L^{p}}+\left\|D^{\beta}\left(\rho(t)-\rho_{p}(t)\right)\right\|_{L^{p}} \leq C \min \left\{1, t^{-\frac{m}{2}\left(1-\frac{1}{p}\right)-|\beta| / 2-1 / 2}\right\}$,

where $\rho_{w}$ and $\rho_{p}$ are respectively the solutions of the $m$-dimensional dissipative wave equation equation and the $m$-dimensional heat equation

$$
\rho_{w, t}+\rho_{w, t t}-\Delta \rho_{w}=0, \rho_{p, t}-\Delta \rho_{p}=0 .
$$

These estimates improve on previous results in [STW03] and [CG04]. 
The Chapman-Enskog expansion in the case $m=1$. For $m=1$, the decay of $u^{2}$ convoluted with the linear kernel and integrated in time gives the same decay estimate of $u$, so we have to keep a nonlinear term in the diffusive expansion. Set $\tilde{A}=\frac{1}{2}\left(L_{0} D_{u_{c}}^{2} f(0)-A_{12}(0)\left(D_{u_{d}} q(0)\right)^{-1} D_{u_{c}}^{2} q(0)\right)$ and $\tilde{B}=A_{12}(0)\left(D_{u_{d}} q(0)\right)^{-1} A_{21}(0)$. We rewrite $(57)$ as

$$
u_{c, t}+\left(A_{11}(0) u_{c}+\tilde{A}\left(u_{c}, u_{c}\right)\right)_{x}+\tilde{B} u_{c, x x}=S_{x},
$$

where $S$ is some given faster decaying term. We replace (59) by the nonlinear diffusive equation

$$
w_{t}+\left(A_{11}(0) w+\tilde{A}(w, w)\right)_{x}+\tilde{B} w_{x x}=0 .
$$

Theorem 8. For $m=1$, let $u_{p}$ be the solution of problem (64), (60), under the assumptions of Theorem 4. For $p \in[1, \infty]$, and a fixed $\mu \in[0,1 / 2)$, if $E_{1}$ sufficiently small with respect to $(1 / 2-\mu)$, then we have

$$
\left\|D^{\beta}\left(u_{c}(t)-u_{p}(t)\right)\right\|_{L^{p}} \leq C \min \left\{1, t^{-\frac{1}{2}\left(1-\frac{1}{p}\right)-\mu-\beta / 2}\right\} F_{\beta+4},
$$

where $C=C\left(\mu, F_{\beta+\sigma}\right)$, for $\sigma$ large enough, where $F_{1}=E_{1}$ and, if $\beta \geq 1$,

$$
F_{\beta+1}= \begin{cases}E_{\beta+1}, & \text { if } p \in[2, \infty], \\ E_{\beta+1}+\left\|D^{\beta} u(0)\right\|_{L^{1}}, & \text { otherwise. }\end{cases}
$$

Example 4. The $p$-system with relaxation. We can apply Theorem 8 to the Example 1. In this case, as already shown in [Ch95], the Chapman-Enskog expansion is given by the semilinear parabolic equation

$$
u_{p, t}+h^{\prime}(0) u_{p, x}+\frac{1}{2} h^{\prime \prime}(0)\left(u_{p}^{2}\right)_{x}-\left(\lambda^{2}-a^{2}\right) u_{p, x x}=0 .
$$

\section{References}

[ABN06] D. Aregba-Driollet, M. Briani, R. Natalini, Asymptotic high-order schemes for dissipative hyperbolic systems, in preparation.

[BRS06] S. Bianchini, B. Hanouzet, R. Natalini, Asymptotic behavior of smooth solutions for partially dissipative hyperbolic systems with a convex entropy, IAC Report 79 (11/2005); to appear in Comm. Pure and Appl. Math.

[CH106] G. Carbou, B. Hanouzet, Comportement semi-linaire d'un systme hyperbolique quasi-linaire: le modle de Kerr-Debye. (French) [Semilinear behavior for a quasilinear hyperbolic system: the Kerr-Debye model] C. R. Math. Acad. Sci. Paris 343 (2006), no. 4, 243-247.

[CH206] G. Carbou, B. Hanouzet, Relaxation approximation of some nonlinear Maxwell initial-boundary value problem. Commun. Math. Sci. 4 (2006), no. $2,331-344$. 
[CLL94] G.-Q. Chen, C.D. Levermore, and T.-P. Liu, Hyperbolic conservation laws with stiff relaxation terms and entropy, Comm. Pure and Appl. Math. 47 (1994), 787-830.

[Ch95] , I.L. Chern, Long-time effect of relaxation for hyperbolic conservation laws, Commun. Math. Phys. 172, (1995), 39-55.

[CG04] J.F. Coulombel and T. Goudon, The strong relaxation limit of the multidimensional isothermal Euler equations, preprint 2004, to appear in Trans. AMS.

[Da00] C. M. Dafermos, Hyperbolic conservation laws in continuum physics, (Springer-Verlag, Berlin, 2000) xvi+443 pp.

[LF71] K. O. Friedrichs and P. D. Lax, Systems of conservation equations with a convex extension, Proc. Nat. Acad. Sci. U.S.A. 68 (1971), 1686-1688.

[Go61] S. K. Godunov, An interesting class of quasi-linear systems, Dokl. Akad. Nauk SSSR 139 (1961), 521-523.

[HH00] B. Hanouzet and Ph. Huynh, Approximation par relaxation d'un système de Maxwell non linéaire, C. R. Acad. Sci. Paris Sér. I Math. 330 (2000), no. 3, 193-198.

[HN03] B. Hanouzet and R. Natalini, Global existence of smooth solutions for partially dissipative hyperbolic systems with a convex entropy, Arch. Ration. Mech. Anal. 169 (2003), no. 2, 89-117.

[Kw87] S. Kawashima, Large-time behaviour of solutions to hyperbolic-parabolic systems of conservation laws and applications, Proc. Roy. Soc. Edinburgh Sect. A 106 (1987), no. 1-2, 169-194.

[Li87] T.-P. Liu, Hyperbolic conservation laws with relaxation, Comm. Math. Phys. 108 (1987), 153-175.

[Na99] R. Natalini, Recent results on hyperbolic relaxation problems, Analysis of systems of conservation laws (Aachen, 1997), Chapman \& Hall/CRC, Boca Raton, FL, 1999, pp. 128-198.

[Ni78] T. Nishida, Nonlinear hyperbolic equations and related topics in fluid dynamics, Département de Mathématique, Université de Paris-Sud, Orsay, 1978, Publications Mathématiques d'Orsay, No. 78-02.

[RS02] T. Ruggeri and D. Serre, Stability of constant equilibrium state for dissipative balance laws system with a convex entropy. Quart. Appl. Math. 62 (2004), no. 1, 163-179.

[SK85] Y. Shizuta and S. Kawashima, Systems of equations of hyperbolic-parabolic type with applications to the discrete Boltzmann equation, Hokkaido Math. J. 14 (1985), no. 2, 249-275.

[STW03] T. Sideris, B. Thomases, and D. Wang, Decay and singularities of solutions of the three-dimensional Euler equations with damping, Comm. Partial Differential Equations 28 (2003), no. 3-4, 795-816.

[Yo04] W.-A. Yong, Entropy and global existence for hyperbolic balance laws, Arch. Ration. Mech. Anal. 172 (2004), no. 2, 247-266.

[Ze99] Y. Zeng, Gas dynamics in thermal nonequilibrium and general hyperbolic systems with relaxation, Arch. Ration. Mech. Anal. 150 (1999), no. 3, 225279. 